well balanced, neither too long nor too short. The chapters are independent so the reader can jump into the book at any point; at the same time, many unifying themes connect the book's different parts.

The figures are exceptional, surpassing those in the previous edition. They do more than summarize the text; they allow the reader to visualize complicated concepts. For example, drawings efficiently communicate the multiprotein interactions in initiation complexes at origins of replication and in DNA

\section{Coffee-table cosmology}

Joseph Silk

The Natural History of the Universe. By Colin A. Ronan. Doubleday/Macmillan Inc: 1991. Pp. 212. £20, \$39.95.

Ancient Light: Our Changing View of the Universe. By Alan Lightman. Harvard University Press: 1991. Pp. 170. \$18.95, £15.25.

Journey Through the Universe. By Jay $M$. Pasachoff. W. B. Saunders: 1991. Pp. 391. £25.95, \$38.75.

Isaac Asimov's Guide to Earth and Space. By Isaac Asimov. Random House: 1991. Pp. 285. \$20.

THE Big Bang theory is our modern mythology, replete with scientific credentials of the highest order. In all environments, from formal banquets and cocktail bars to academic classrooms and funding agencies, it provides an endless source of debate and inspiration. Popular books continue to appear that attempt to communicate the fascination and excitement of the quest for our origins that underlies the science of cosmology.

Ronan's The Natural History of the Universe is a glossy coffee-table book that is a worthy addition to science popularization. An enthusiastic amateur astronomer, Ronan has generated a beautifully produced and illustrated sequence of thumbnail sketches encompassing the entire scheme of cosmic evolution. This splendid overview cannot fail to impress, despite occasional flaws in detail. Professional astronomers are woefully derelict in their duty to popularize, yet would not have been quite so vulnerable as Ronan to the latest press releases. Recent discoveries of low-mass stars have failed to provide any indication of the nature of "missing" mass. Nor would any astronomer tolerate "unknown numbers of black holes of unknown mass" as an alternative. One can only become more mystified as to what 'texture' might be after reading Ronan's NATURE · VOL $355 \cdot 30$ JANUARY 1992 elongation complexes at replication forks. By comparison to the first edition, the figures alone show how much clearer the mechanisms of DNA replication have become in the past decade.

My only regret is that the expansion of the book has rendered Kornberg's personal vision less apparent, although thankfully his well-known love of enzymes still shines through everywhere. $\square$

Sue Wickner is in the Laboratory of Molecular Biology, National Institutes of Health, Bethesda, Maryland 20892, USA.

book (it's a kind of fossil froth caused by the Big Bang), and it is not true that inflation explains the observed ratio of photons per baryon. But these are quibbles: let the reader judge.

In Ancient Light, Lightman demonstrates the reliability of a professional astrophysicist. Inevitably this slender account of the Big Bang theory is more technical than Ronan's book, but is enlivened by portraits of the leading characters. Due credit is gratefully given to the many creators of inflationary cosmology, although Andrei Sakharov is unaccountably omitted as the godfather of baryon synthesis. We learn that the low entropy inferred before inflation at the beginning of the Universe is about as unnatural as finding the outcome of wiring one's favourite pet to a computer terminal to be a Beethoven sonata. But we also know that the quantum gravity theory of the first $10^{-43}$ seconds of the Universe is so woefully inadequate that any extrapolation of the second law of thermodynamics - that entropy never decreases - becomes highly dubious. This is more a flaw in our fundamental physics than in the Big Bang theory.

For the briefest instant, lasting less than $10^{-30}$ seconds, the very early Universe underwent a dramatic increase in its rate of expansion. Explaining inflation provides an acid test that few popularizations pass. Lightman talks about a false vacuum that acts like negative gravity, repelling matter. Ronan tells us that the false vacuum forms when supercooling occurs (in fact, it was always present, but only briefly did it come to dominate the pressure). One could circumvent some of the jargon by noting that cooling as the Universe underwent a phasechange temporarily results in a negative pressure as regions form of the new phase: low-energy examples are condensation of steam, freezing of water and formation of crystals. Negative pressure is negative energy density, which is negative mass, which in turn is antigravity: hence repulsion. This is what drives inflation. The mystery, still unsolved, is why the pressure remained negative long enough to generate the enormous degree of inflation that is required, followed by the relatively smooth reheating of the now supercooled Universe to reproduce the hot Big Bang that is a cherished article of faith among cosmologists.

Those looking for a more academic approach to the story of everything will benefit from Pasachoff's Journey Through the Universe. Pasachoff has distilled the cosmic essence from his elementary astronomy textbook. The result is a lavishly illustrated and scientifically honest description of contemporary astronomy and contemporary astronomers. Isaac Asimov's Guide to Earth and Space takes an alternative tack. Asimov poses more than one hundred questions that span properties of the Earth to stars and galaxies, culminating in the usual "Where are we going?" and "Where did we come from?" His answers are concise and nontechnical, a boon for the reader in a hurry. Yet when on unfamiliar ground - inflation again - he too pays incoherent tribute to fluctuations in a false vacuum.

None of these attempts at popularization need disgrace a scientist's bookshelf. All are carefully researched and well written. Perhaps the touch of inspiration is lacking, but the intended audience of interested but scientifically semiliterate readers will surely benefit. $\square$

Joseph Silk is in the Departments of Astronomy and Physics, University of California, Berkeley, California 97420, USA.

\section{Methods books}

- There are two new introductions to the handling of numerical data: A Practical Guide to Data Analysis for Physical Science Students by Louis Lyons (Cambridge University Press, price £19.95, \$34.95 (hbk), $£ 7.97, \$ 12.95(\mathrm{pbk}))$ and Basic Statistics for Laboratories: A Primer for Laboratory Workers by W. D. Kelly, T. A. Ratliff Jr and C. Nenadic (Van Nostrand Reinhold, price \$44.95)

- Radioisotopic Methods for Biological and Medical Research by H. W. Knoche is published by Oxford University Press, price $£ 30$. - The Handbook of Infrared and Raman Characteristic Frequencies of Organic Molecules by D. Lin-Vien, N. B. Colthup, W. G. Fateley and J. G. Grasselli is sure to become a standard reference work for every practicing spectroscopist. Published by Academic, price $\$ 89.95$.

- IRL Press at Oxford University Press have issued two new volumes in their successful Practical Approach Series. Essential Molecular Biology, edited by T. A. Brown, covers the procedures needed to identify specific genes and study their structure and cellular expression. Oligonucleotides and Analogues, edited by $F$. Eckstein, will be of interest to academic and industrial researchers. Each is issued in paperback at $£ 22.50$, but is also available as a spiral-bound hardback.

- New in paperback is A Handbook of Silicate Rock Analysis by P. J. Potts. Published by Blackie, price is $£ 45$. For a review see Nature 327, 198 (1987). 